ARTICLE

Received 22 Oct 2012 | Accepted 8 Feb 2013 | Published 19 Mar $2013 \quad$ DOl: 10.1038/ncomms2588

\title{
Magnetic spin imaging under ambient conditions with sub-cellular resolution
}

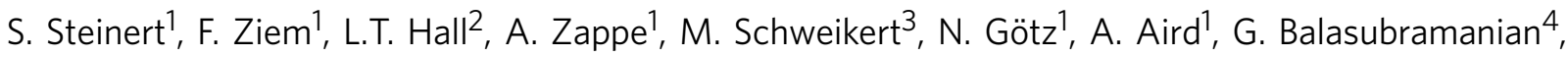 \\ L. Hollenberg ${ }^{2} \&$ J. Wrachtrup ${ }^{1}$
}

The detection of small numbers of magnetic spins is a significant challenge in the life, physical and chemical sciences, especially when room temperature operation is required. Here we show that a proximal nitrogen-vacancy spin ensemble serves as a high precision sensing and imaging array. Monitoring its longitudinal relaxation enables sensing of freely diffusing, unperturbed magnetic ions and molecules in a microfluidic device without applying external magnetic fields. Multiplexed charge-coupled device acquisition and an optimized detection scheme permits direct spin noise imaging of magnetically labelled cellular structures under ambient conditions. Within $20 \mathrm{~s}$ we achieve spatial resolutions below $500 \mathrm{~nm}$ and experimental sensitivities down to 1,000 statistically polarized spins, of which only 32 ions contribute to a net magnetization. The results mark a major step towards versatile subcellular magnetic imaging and real-time spin sensing under physiological conditions providing a minimally invasive tool to monitor ion channels or haemoglobin trafficking inside live cells.

\footnotetext{
${ }^{1}$ 3rd Institute of Physics and Research Center SCOPE, University Stuttgart, Stuttgart 70569, Germany. ${ }^{2}$ Centre for Quantum Computation and Communication Technology, School of Physics, University of Melbourne, Parkville, Victoria 3010, Australia. ${ }^{3}$ Biologisches Institut, University Stuttgart, Stuttgart 70569, Germany. ${ }^{4}$ Research Group Nanoscale Spin Imaging, Max-Planck Institute of Biophysical Chemistry and Center for Nanoscale Microscopy and Molecular Physiology of the Brain (CNMPB), Goettingen 70377, Germany. Correspondence and requests for materials should be addressed to S.S. (email: s.steinert@physik.uni-stuttgart.de).
} 
mprovements to current magnetic resonance imaging (MRI) techniques will allow chemical analysis of samples with high resolution down to sub-cellular volumes. However, conventional magnetic imaging is hindered by inductive detection requiring a sufficient magnetic flux from the sample to induce a detectable current in the pickup coil. Increases in spatial resolution reduce the number, $n$, of detected spins until the statistical polarization, whose magnitude is of order $\sqrt{n}$, exceeds the magnetization due to Boltzmann polarization. The resultant vanishing mean field strength and its stochastic nature render common detection schemes ineffective. Precision magnetometers provide exceptional sensitivities reaching a few $\mathrm{fT} / \sqrt{ } \mathrm{Hz}^{2-5}$, yet their relatively large size and/or operational conditions prohibit sensing of small numbers of spin magnetic moments at ambient conditions and sub-micron resolution. Sensors based on nitrogen-vacancy (NV) centres in diamond are promising magnetometers because of their atomic size. This allows placement of the sensor with few nanometre proximity to the sample while retaining superior volume-to-sensitivity scaling, room temperature operation and optical readout ${ }^{6-8}$. Precision metrology has already been demonstrated for DC and AC fields ${ }^{9-14}$, spins within the diamond lattice ${ }^{15-18}$ and surface spins ${ }^{19}$. Here, we demonstrate sensing and imaging of stochastic magnetic fluctuations originating from freely diffusing electron spins such as paramagnetic oxygen $\left(\mathrm{O}_{2}, S=1\right)$, $\mathrm{MnCl}_{2}(S=5 / 2)$ and Gadolinium ions $\left(\mathrm{Gd}^{3+}, S=7 / 2\right)$ in liquids, immobilized in polymers and linked specifically to cellular structures.

\section{Results}

NV relaxometry. In low external magnetic fields, freely diffusing ions exhibit a zero-mean field, but magnetic field fluctuations due to statistical spin polarization cause a non-zero RMS field $\sqrt{\left\langle B^{2}\right\rangle}>0$ with random phase. Such stochastic fields are difficult to detect, but the NV offers a striking avenue to measuring such random fields by monitoring its quantum relaxation ${ }^{20,21}$. To benefit from ensemble sensing sensitivity 22,23 , we employ an array of atomic sized NV sensors $\left(\sim 1,000 \mu \mathrm{m}^{-2}\right)$ with a calculated mean depth of $h=6.7 \mathrm{~nm}$ (ref. 24), as illustrated in Fig. 1. After preparation of a distinct NV spin state, interaction with the environment leads to $\mathrm{NV}$ relaxation with rate $\Gamma=\Gamma_{\text {int }}+\Gamma_{\text {env }}$. The contributions intrinsic to the diamond, $\Gamma_{\text {int }}$, include spin impurities and vibrational lattice dynamics, whereas $\Gamma_{\text {env }}$ depends on the external environment to which the system can deliberately be exposed. Relaxation may occur via the transverse and longitudinal relaxation channels characterized by their respective decay times $T_{2}$ and $T_{1}$. The transverse dephasing rate $\Gamma_{2}=1 / T_{2}$ is increased by fluctuations at low frequencies $(\mathrm{kHz}$ to $\mathrm{MHz}$ ) and can be monitored using spin echoes or higher order dynamical decoupling microwave control protocols ${ }^{25-27}$. The longitudinal relaxation rate $\Gamma_{1}=1 / T_{1}$ describes the population decay of a polarized spin into thermal equilibrium. It is susceptible to frequencies at the NV Larmor precession $\omega_{0}=D \pm \gamma B_{0}$, where $\gamma$ is the gyromagnetic ratio and $B_{0}$ is a magnetic offset field. Owing to the large NV zero-field splitting of $D=2.87 \mathrm{GHz}$ this is in the $\mathrm{GHz}$ range at low fields.

Spin noise sensing. First, we demonstrate magnetic sensing of various chemical environments by probing both relaxation channels along one preferred $\mathrm{NV}$ axis $\left(B_{0}=5 \mathrm{mT}\right)$ in the presence of air, water and a solution of $1 \mathrm{M} \mathrm{Gd}^{3+}$ (see Table 1). Particularly the large magnetic moment of $\mathrm{Gd}^{3+}$ has made the $\mathrm{Gd}^{3+}$ chelate a prime candidate as relaxation contrast agent in $\mathrm{MRI}^{28}$. In this system, probing $\Gamma_{2}$ by a Hahn echo sequence shows only marginal changes in $T_{2}$ for $\mathrm{Gd}^{3+}$, while multi-pulse CPMG81 is mildly responsive with a change in $T_{2}$ of $13.5 \%$. The longitudinal $T_{1}$, however, exhibits a prominent reduction of $94 \%$ in the presence of $\mathrm{Gd}^{3+}$ and was similarly responsive to dissolved $\mathrm{MnCl}_{2}$ and with $\mathrm{O}_{2}$ saturated water (Fig. $2 \mathrm{a}$ and Supplementary Fig. S1). Focusing on the dominant $\mathrm{Gd}^{3+}$, the relaxation rate $\Gamma_{\mathrm{Gd}}$ induced by freely diffusing $\mathrm{Gd}^{3+}$ depends on the corresponding RMS magnetic field and its spectral density $S_{\mathrm{Gd}}(\omega)$. For a Gaussian process, the latter is given by $S_{\mathrm{Gd}}(\omega)=\sqrt{2 / \pi} \cdot f_{\mathrm{Gd}} /\left[\left(\omega-\omega_{0}\right)^{2}+f_{\mathrm{Gd}}^{2}\right]$, where $\omega_{0}$ is the Larmor frequency of $\mathrm{Gd}^{3}+$. Boltzmann polarization and $\omega_{0}$ can be neglected for the low $B_{0}$ fields applied. $S_{\mathrm{Gd}}(\omega)$ is instead dominated by statistical polarization and substantial broadening effects of zero-mean fluctuations $f_{\mathrm{Gd}}=f_{\text {dipole }}+$ $f_{\text {vib }}+f_{\text {trans }}+f_{\text {rot }}$. The contribution from the concentrationdependent dipole coupling between the $\mathrm{Gd}^{3+}$ is given by

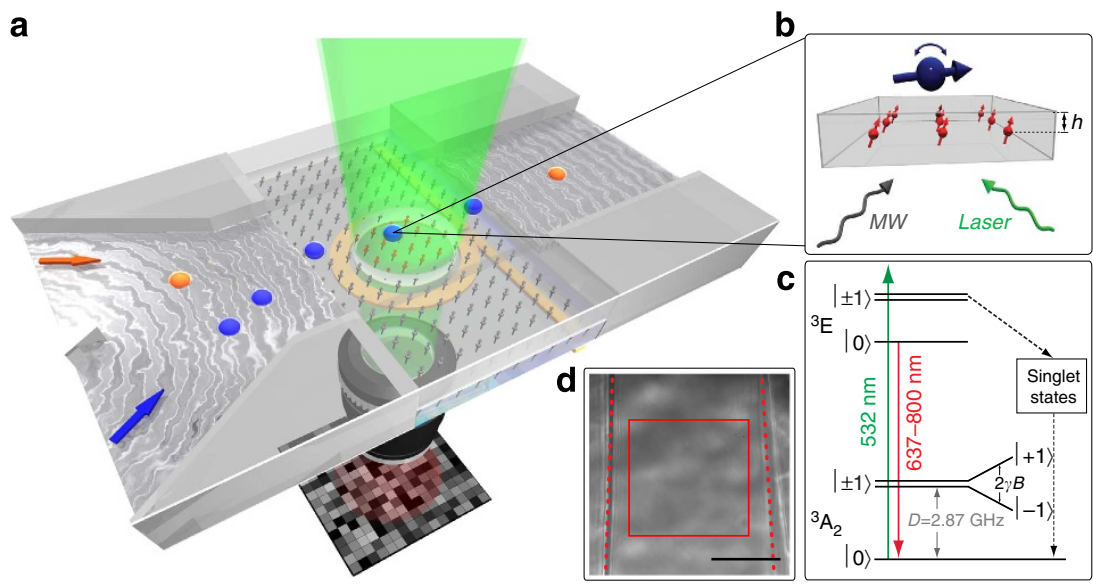

Figure 1 | Widefield magnetometry with microfluidic detection. (a,b) Widefield excitation (green) of the NV spin array (red arrows) and spatially resolved CCD detection of NV fluorescence. Homogeneous spin manipulation of the NV array is achieved via a lithographic $\Omega$-microwave structure (yellow). $\mathrm{Gd}^{3+}$ ions (blue spheres in $\mathbf{a}, \mathbf{b}$ in aqueous solution are introduced using a microfluidic channel placed directly on top of the sensor proximal to the NV array with $h=6.7 \mathrm{~nm}$. (c) Energy level scheme of NV centre illustrating the high fluorescent signal extending from 637-800 nm. The $|0\rangle$ spin sublevel has a stronger fluorescence than the $| \pm 1\rangle$ states as they emit less photons owing to a higher probability to enter the long-living singlet states. NV relaxation is thus readout optically. (d) Brightfield image of the microfluidic channel with channel boundaries (dotted lines) and $30 \times 30 \mu m^{2}$ detection region of interest (ROI, red rectangle). Scale bar, $20 \mu \mathrm{m}$. 
$f_{\text {dipole }}=c_{\mathrm{Gd}} \cdot 77 \mathrm{GHz} \mathrm{M}^{-1}$ (Supplementary Note 1 ), where $c_{\mathrm{Gd}}$ is the spin concentration in $\mathrm{moll}^{-1}$. Intrinsic vibrational spin relaxation of the complexed $\mathrm{Gd}^{3+}$ ion yields a constant fluctuation of $f_{\text {vib }} \sim 50 \mathrm{GHz}^{29}$, while rotational motion $f_{\text {rot }}$ and translational diffusion $f_{\text {trans }}$ cause fluctuations of $\sim 140 \mathrm{MHz}$ (Supplementary Fig. S2). The resulting broadening of the spectral density $S_{\mathrm{Gd}}(\omega)$ is therefore effectively constant up to a few tens of $\mathrm{GHz}$ (Fig. 2b), where resonant $\mathrm{Gd}^{3+}$-induced fluctuations $B_{\mathrm{x}}$ and $B_{\mathrm{y}}(\mathrm{NV}$ axis defines $\mathrm{z})$ cause $\Gamma_{1}$ relaxation of the $\mathrm{NV}$.

\section{Table 1 | Transverse $\left(T_{2}\right)$ and longitudinal $\left(T_{1}\right)$ NV spin relaxation in various environments.}

\begin{tabular}{|c|c|c|c|}
\hline & $T_{2, \text { Hahn }}$ & $T_{2, \text { CPMG81 }}$ & $T_{1}$ \\
\hline Air ( $\mu \mathrm{s})$ & $1.92 \pm 0.04$ & $42.9 \pm 1.8$ & $1,155 \pm 87$ \\
\hline $\mathrm{dH}_{2} \mathrm{O}(\mu \mathrm{s})$ & $1.91 \pm 0.03$ & $42.1 \pm 2.1$ & $906 \pm 75$ \\
\hline $\mathrm{Gd}^{3}+1 \mathrm{M}(\mu \mathrm{s})$ & $1.85 \pm 0.05$ & $37.1 \pm 2.0$ & $70 \pm 4$ \\
\hline$\Delta \mathrm{T}(\%)$ & 3.7 & 13.5 & 94.0 \\
\hline $\mathrm{SNR}=\Gamma_{\mathrm{Gd}} / \Gamma_{\mathrm{int}}$ & 0.04 & 0.16 & 15.5 \\
\hline
\end{tabular}

NV, nitrogen vacancy.

The flow cell was filled with either air, ultrapure water or $1 \mathrm{M} \mathrm{Gd}{ }^{3+}$. The change $\Delta T$ in the corresponding relaxation times is given by $\Delta T=\left(T_{\text {air }}-T_{\mathrm{Gd}}\right) / T_{\text {air }} \Gamma_{\mathrm{Gd}}=1 / T_{\mathrm{Gd}}-1 / T_{\text {air }}$ corresponds to the induced relaxation in the presence of $1 \mathrm{M} \mathrm{Gd}{ }^{3}+$ and $\Gamma_{\text {int }}=1 / T_{\text {air }}$ is the diamond intrinsic relaxation. As $\Gamma_{\text {int }}$ competes with the $\mathrm{Gd}^{3+}$-induced relaxation, the relaxometric signal-to-noise ratio is given by $\mathrm{SNR}=\Gamma_{\mathrm{Gd}} / \Gamma_{\text {int }}$. Errors, $1 \sigma$ statistical uncertainty.
The magnetic field variance is derived from the dipolar NV$\mathrm{Gd}^{3}+$ coupling and is given by

$$
\left\langle B_{\mathrm{Gd}}^{2}\right\rangle=\left\langle B_{\mathrm{x}}^{2}\right\rangle+\left\langle B_{\mathrm{y}}^{2}\right\rangle=\frac{21 \cdot 10^{3} \pi N_{\mathrm{A}} c_{\mathrm{Gd}}}{16 h^{3}}\left(\frac{\mu_{0} \hbar}{4 \pi} \gamma_{\mathrm{NV}} \gamma_{\mathrm{Gd}}\right)^{2},
$$

where $N_{\mathrm{A}}$ is the Avogadro constant, $\mu_{0}$ the vacuum permeability, $h$ the mean depth of the NV centres and $\gamma_{\mathrm{NV}} \approx \gamma_{\mathrm{Gd}}$ (Supplementary Fig. S4). The effective RMS field strength is then given by $B_{\mathrm{rms}}=\sqrt{\left\langle B_{\mathrm{Gd}}^{2}\right\rangle} / \gamma_{\mathrm{NV}}$. The time-dependent probability of finding the $\mathrm{NV}$ in the $|0\rangle$ state (Supplementary Note 2) is given by

$$
P_{0}(\tau)=\frac{1}{6}\left(2+e^{-\Gamma_{1}^{-} \tau}+e^{-\Gamma_{1}^{+} \tau}+2 e^{-\left(\Gamma_{1}^{-}+\Gamma_{1}^{+}\right) \tau}\right),
$$

where $\Gamma_{1}^{ \pm}$are the individual decay rates of each sensitivity window $F_{1}^{ \pm}$(Fig. 2b) integrated over $S_{\mathrm{Gd}}(\omega)$. The overall decay rate $\Gamma_{1, \mathrm{Gd}}$ of the longitudinal $\mathrm{NV}$ magnetization is then given by

$$
\Gamma_{1, \mathrm{Gd}}=-\left.\frac{d}{d \tau}\right|_{\tau=0} P_{0}(\tau) \approx 2 \frac{f_{\mathrm{Gd}}\left\langle B_{\mathrm{Gd}}^{2}\right\rangle}{f_{\mathrm{Gd}}^{2}+D^{2}} .
$$

This theoretical prediction was experimentally verified by varying $c_{\mathrm{Gd}}$ (Fig. 2c), showing excellent agreement for the nontrivial dependence on $c_{\mathrm{Gd}}$. At nanometre length scales, the a
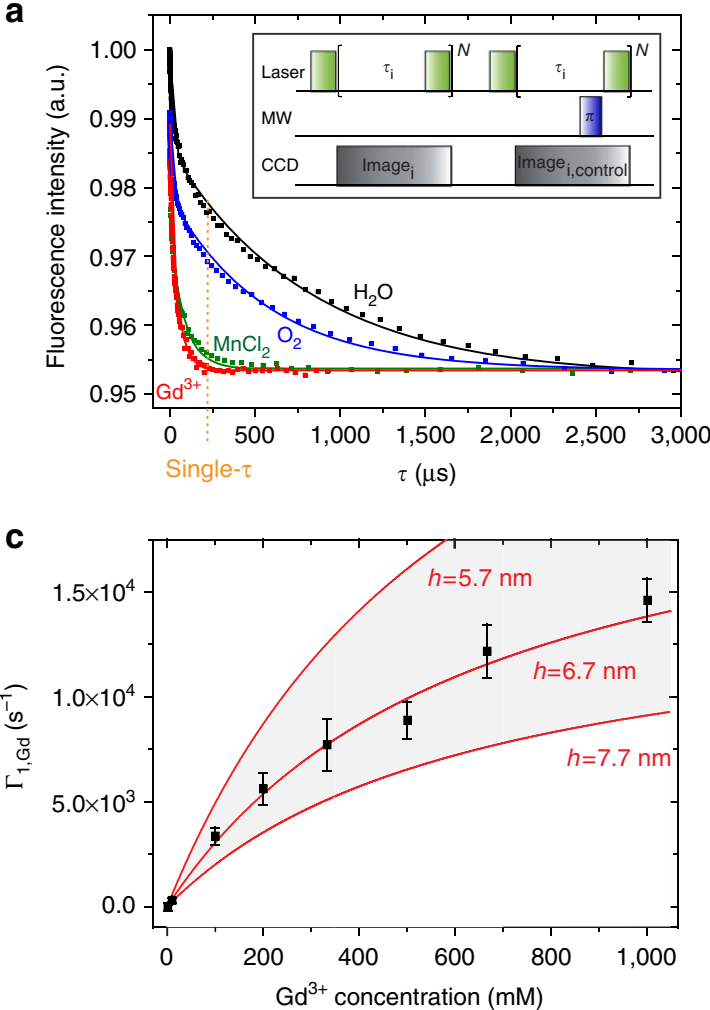

b
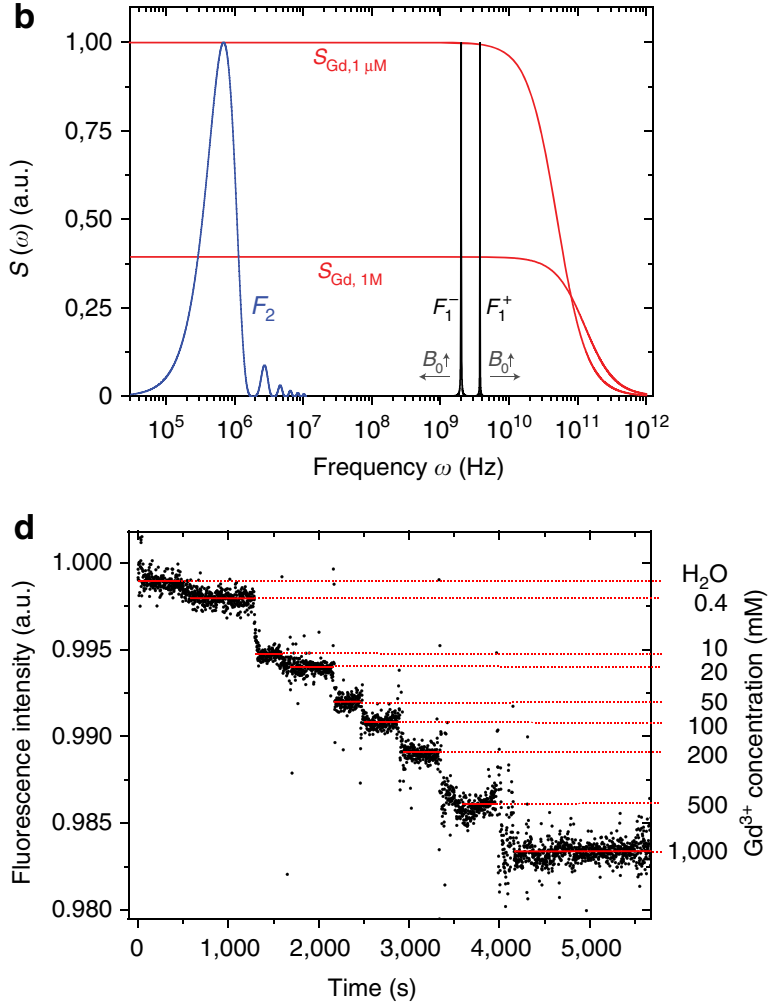

Figure 2 | Microfluidic spin sensing. (a) $T_{1}$ relaxation curves of the NV ensemble in the presence of water (black), oxygenated water ( $\sim 1.5 \mathrm{mM}$, blue), $1 \mathrm{M} \mathrm{MnCl}_{2}$ (green) and $1 \mathrm{M} \mathrm{Gd}^{3+}$ (red). Solid curves are bi-exponential fits to the data. Optimized spin detection via direct fluorescent readout is achieved at a discrete interrogation period $\tau$ (orange). The inset shows the measurement pulse sequence and normalization. (b) Spectral density $S_{G d}(\omega)$ of $G_{d}{ }^{3+}$ for two distinct concentrations (red curves) illustrating the broadening effect due to $\mathrm{Gd}^{3+}$ coupling $\left(f_{\text {dipole }}\right.$ ) at higher concentrations. Although the sensitivity windows of $T_{2}$-decoherence $\left(F_{2}\right.$, blue) is limited to low $\mathrm{MHz}$ fluctuations, $T_{1}$-relaxometry allows to probe a wide frequency range up to $\mathrm{GHz}$ with two sensitivity windows $\left(F_{1}^{-}\right.$and $F_{1}^{+}$, black). $F_{1}^{ \pm}$can be Zeeman-shifted via $B_{0}$ enabling experimental detection of $S_{G d}(\omega)$. (c) Experimental relaxation rate $\Gamma_{1, G d}$ as a function of the $\mathrm{Gd}^{3+}$ concentration (black squares with $1 \sigma$ s.e. and three independent measurements) and analytical predictions for specific mean implantation depths $h$ (red curves). (d) Dynamic microfluidic single- $\tau$ detection $\left(\tau=100 \mu \mathrm{s}\right.$ ) of varying $\mathrm{Gd}^{3+}$ concentrations with a single data point acquisition time of $t_{\mathrm{m}} \sim 2 \mathrm{~s}$. 
sample-sensor distance is a key parameter as $\Gamma_{1, \mathrm{Gd}} \propto\left\langle B_{\mathrm{Gd}}^{2}\right\rangle \propto$ $h^{-3}$ and thus represents a viable tool to access the average implantation depth of nitrogen ions (Fig. 2c). The relaxometric $\mathrm{SNR}=\Gamma_{\mathrm{Gd}} / \Gamma_{\text {int }}$ of $T_{1}$ is much higher as $\Gamma_{2 \text {,int }} \gg \Gamma_{1 \text {,int }}$ due to the low spin-orbit coupling accompanied by a low phonon density in diamond ${ }^{30}$. Hence, in this system longitudinal $T_{1}$ outperforms $T_{2}$-relaxometry by two orders of magnitude (Supplementary Fig. S3) and is employed from here on.

Microfluidic single- $\tau$ detection. Next, we optimized the microfluidic detection by converting the $T_{1}$ signal directly into a measureable fluorescence. Instead of detecting the full relaxation curve, the fluorescent $\mathrm{NV}$ response at a single- $\tau$ point is utilized as direct concentration readout (Fig. 2a). Maximum sensitivity is achieved at $\tau \sim T_{1, \mathrm{Gd}} / 2$ for $\mathcal{c}_{\mathrm{Gd}}<10 \mathrm{mM}$ and approaches $T_{1, \mathrm{Gd}}$ for higher $c_{\mathrm{Gd}}$ (Supplementary Note 4 and Supplementary Figs S6-S8). Therefore, varying $\mathrm{Gd}^{3+}$ concentrations can be monitored dynamically with temporal resolutions in the order of a second (Fig. 2d). For maximal sensitivity all four crystallographic NV orientations can be employed, as $\left\langle B_{\mathrm{Gd}}^{2}\right\rangle$ exhibits a random magnetic field orientation. This is achieved by measuring in near-zero field ( $50 \mu \mathrm{T}$ earth field) and probing all NV orientations at $D=2.87 \mathrm{GHz}$, which effectively improves the fluorescent single- $\tau$ contrast and increases the number of sensing NV spins by a factor of four (Supplementary Fig. S9). We applied a statistical $t$-test $(2 \sigma)$ to the fluorescence traces to identify the lowest concentration $c_{\mathrm{Gd}}^{\mathrm{min}}$ still significantly different to pure water. After a total measurement time of $t_{\mathrm{m}}=20 \mathrm{~s}$ and an optimized $\tau=400 \mu$ s, we resolved $c_{\mathrm{Gd}}^{\mathrm{min}}=250 \mu \mathrm{M}$ and $80 \mu \mathrm{M}$ for single and all four NV axes, respectively. In addition, the multiplexed charge-coupled device (CCD) detection allows varying the detection voxel equivalent to an effective change in spatial resolution of magnetic sensing. For fixed $t_{\mathrm{m}}$ and CCD detection, $c_{\mathrm{Gd}}^{\min }$ depends on the shot noise of detected photons and thus on the applied pixel binning (Supplementary Fig. S3). Although single pixel analysis is feasible, the lower limit of the spatial resolution for the widefield detection is $\Delta r_{x y} \sim 430 \mathrm{~nm}$ (Supplementary Fig. S10). In our experimental setup this translates into $4 \times 4$ pixels (equivalent to $\Delta r_{x y} \sim 460 \mathrm{~nm}$ ). For such a small voxel, we detected $c_{\mathrm{Gd}}^{\min }=500 \mu \mathrm{M}$, which approaches typical ion concentrations encountered in oxidative bursts ${ }^{31}$ or senile placques ${ }^{32}$. As demonstrated previously, lower spin concentrations can conveniently be sensed by spatially averaging over larger voxels. To determine the sensitivity in terms of number of spins per detection voxel $n_{\mathrm{Gd}}=c_{\mathrm{Gd}}^{\mathrm{min}} \cdot 10^{3}$. $N_{\mathrm{A}} \cdot \Delta r_{\mathrm{xy}}^{2} \cdot \Delta r_{z}$ we emphasize that $\Gamma_{1, \mathrm{Gd}}$ integrates the signal from the spins above the surface while proximal ions $(r=h)$ contribute most due to the $h^{-3}$ scaling. We determined the height dependence by employing polymer spacers between NV and $\mathrm{Gd}^{3+}$, which yielded a decay of $\Gamma_{1, \mathrm{Gd}}(r)$ to $1 / \mathrm{e}$ at $\Delta r_{\mathrm{z}}=15 \mathrm{~nm}$ (Supplementary Fig. S11). Taking the experimental sensitivity to $c_{\mathrm{Gd}}^{\mathrm{min}}=500 \mu \mathrm{M}$ detected in $t_{\mathrm{m}}=20 \mathrm{~s}$ and a spatial resolution of $\Delta r_{\mathrm{xy}}=460 \mathrm{~nm}(4 \times 4$ pixels $)$, this corresponds to an experimental detection of 1,000 statistically polarized spins of which only 32 ions contribute to an effective net magnetization. This spin sensitivity is an improvement by more than four orders of magnitude compared with other state-of-the-art magnetic sensing techniques operating at ambient conditions ${ }^{33-35}$. Single spin detection has been demonstrated using magnetic resonance force microscopy $y^{3,8}$, but at the expense of cryogenic temperatures and significantly longer acquisition times. The combination of spatio-temporal resolution, spin sensitivity and the operability under ambient conditions using widefield NV relaxometry paves the way towards minimally invasive real-time observation of chemical and biological processes involving magnetic spins on the sub-cellular level.
Relaxometric imaging. Finally, this unprecedented spatialtemporal resolution is used to image magnetic fluctuations originating from samples on the sensor array (Fig. 3a). A periodic grid of lithographically patterned $\mathrm{Gd}^{3}+$ is easily resolvable in the reconstructed $T_{1}$ image (Fig. $3 \mathrm{~b}$ ). However, $t_{\mathrm{m}}$ can be reduced by orders of magnitude applying the optimized single- $\tau$ detection, yielding yet higher contrast of magnetic imaging (Fig. 3c). For high-resolution magnetic imaging in biological samples, we have specifically labelled the plasma membrane of HeLa cells with caged $\mathrm{Gd}^{3}+$ ions and an Alexa532 fluorophore via biotinylated poly-L-lysine. As the NV sensor is most sensitive to proximal spins, we placed $150 \mathrm{~nm}$ thin ultramicrotome sections of labelled cells onto the diamond sensor. The control fluorophore indicates a successful label of the magnetic marker to HeLa cells (Fig. 3d), while the boundary of the cell is clearly present in the magnetic image (Fig. 3e). Furthermore, a line scan through the magnetically labelled plasma membrane verifies a spatial resolution of $\Delta r_{x y}=472 \mathrm{~nm}$ approaching the inherent optical limit of the conventional widefield technique. A complete simulation of the single- $\tau$ detection based on shot noise limited photon detection agrees with the experimental sensitivities (Fig. 4, Supplementary Fig. S8). The key advantage of magnetic spin labels is the potential for chemically selective spin contrast imaging as each spin label is expected to have a distinct $S(\omega)$, for example, by binding to a certain biological complex. Using the NV, the spectral density can be measured with a high bandwidth as $\Gamma_{1}$ provides a narrow and via $B_{0}$ tunable sensitivity window ( $\mathrm{F}_{1}$ in Fig. $2 \mathrm{~b}$, experimental realization in Supplementary Note 3 and Supplementary Fig. S5).

\section{Discussion}

The results presented here demonstrate highly sensitive minimally invasive spin sensing and imaging of unperturbed electron spins at room temperature. We have shown the NV-based relaxometric technique offers several advantages over other techniques capable of sensing statistical polarization ${ }^{3,33,35,36}$ as it is operable under ambient conditions with no requirements of strong magnetic fields or radiofrequency pulses. Quantum relaxation of the NV sensor has the potential to emerge as a novel technique of high-throughput analytical sciences and contrast-enhanced optical-MRI at the nanoscale. A considerable improvement in sensitivity is possible by decreasing the $\mathrm{NV}$ depth $h$ and the voxel size $\Delta r_{\mathrm{xy}}$ equivalent to enhancing the spatial resolution (Fig. 4 and Supplementary Fig. S8). With $h=2.5 \mathrm{~nm}$ and typical resolutions of $\Delta r_{\mathrm{xy}}=50 \mathrm{~nm}$ for structured widefield illumination ${ }^{37}$, a sensitivity in the order of ten $\mathrm{Gd}^{3+}$ spins is expected. At the expense of longer integration, scanning techniques such as stimulated emission depletion would boost $\Delta r_{\mathrm{xy}}$ down to $8 \mathrm{~nm}^{38}$ reaching even single spin sensitivities. Alternatively to our ensemble sensor, local spin densities could be monitored even inside living cells by employing single NVs embedded in nanodiamonds ${ }^{39,40}$. The high temporal resolution of widefield magnetometry favors sub-cellular visualization also of label-free dynamic processes, for instance the production of free radicals in cell death, the regulation of homoeostasis through ion channels ${ }^{41}$ or haemoglobin trafficking by imaging paramagnetic oxygen.

\section{Methods}

Experimental setup. ${ }^{15} \mathrm{~N}_{2}$ at a fluence of $10^{13} \mathrm{~cm}^{-2}$ with an energy of $4 \mathrm{keV}$ per atom was homogeneously implanted into a $80-\mu \mathrm{m}$ thin ultrapure type-IIa diamond (Element6) yielding a density of sensing NV spins of $\sim 1,000 \mu \mathrm{m}^{-2}$. According to a SRIM simulation (stopping and range of ions in matter) ${ }^{24}$, the mean depth of the $\mathrm{NV}$ sensor is $h=6.7 \pm 2.8 \mathrm{~nm}$. A $500 \mathrm{MHz}$ PulseBlaster card (ESR-Pro-II, Spincore) was employed for timing the triggering of microwave pulses, CCD integration and the laser excitation. Laser (1.5 W of a 532- $\mathrm{nm} \mathrm{cw}$; Verdi, Coherent) was directed through an AOM (Crystal Technology) and focused onto the backfocal plane of a $60 \times$ oil objective, $1.49 \mathrm{NA}$ (Olympus). An $800 \mathrm{~ns}$ laser pulse allows 
a

$\mathrm{Gd}^{3+}$ labeled structures

i.e. polymers, biological cells

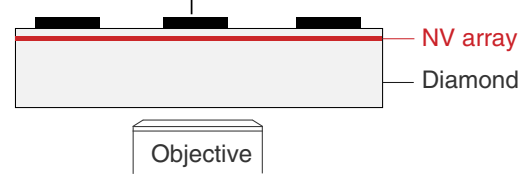

Objective b

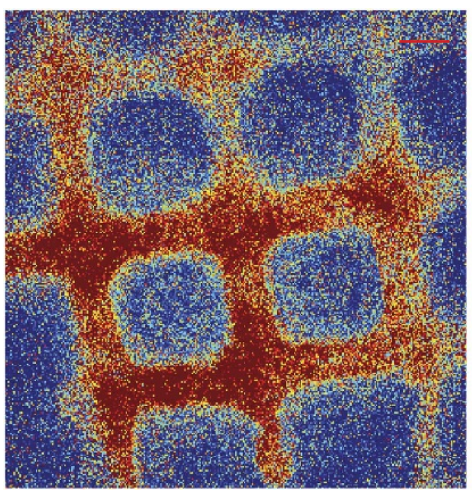

e

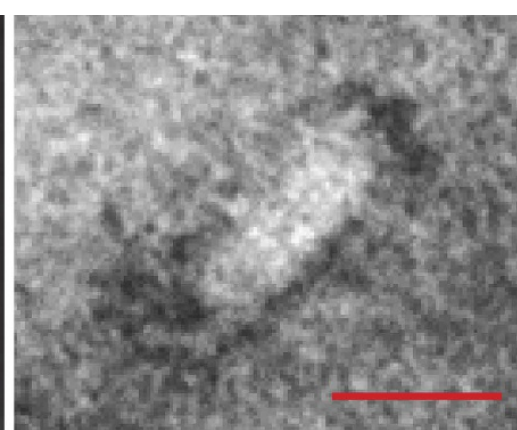

135

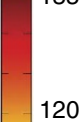

120

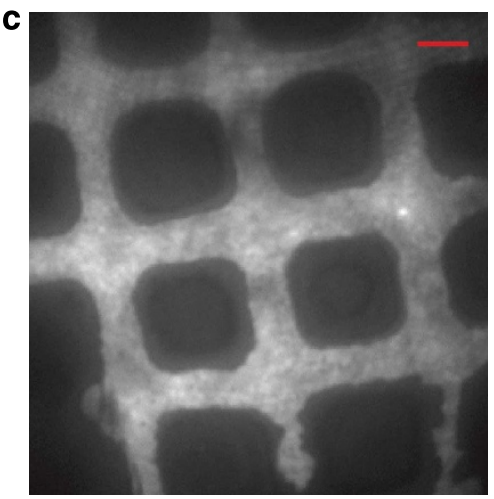

d

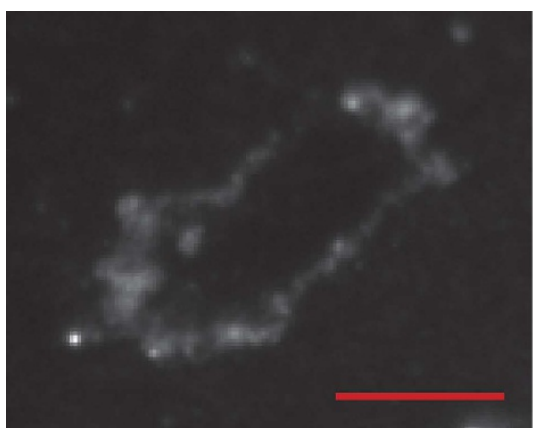

f

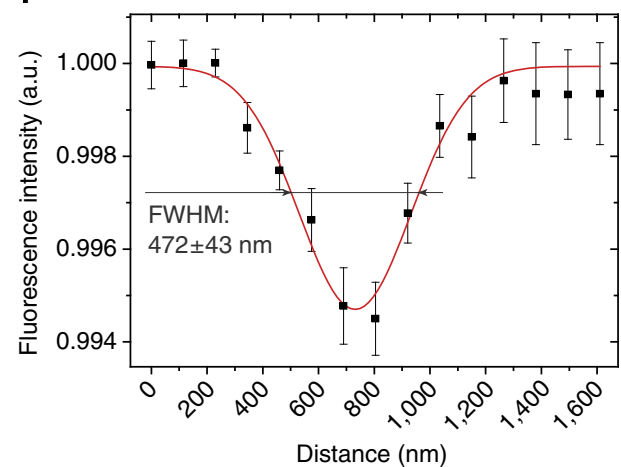

Figure 3 | Spin contrast imaging. (a) Schematic of magnetic spin imaging. (b) $T_{1}$ weighted image of lithographically patterned $\mathrm{Gd}^{3+}$ grid (blue rectangular regions with low $T_{1}$ ) on top of diamond sensor. Three data sets, each containing the full $T_{1}$-decay information with varying $\tau$, were acquired within $t_{\mathrm{m}}=45 \mathrm{~min}$, subsequently averaged and fitted to obtain $T_{1}$ for each pixel. (c) Single- $\tau$ imaging $(\tau=150 \mu \mathrm{s})$ directly yields dark areas where $\mathrm{Gd}^{3}+$ is present due to the increased NV relaxation. Although a single image $\left(t_{m}=2 \mathrm{~s}\right)$ is sufficient to identify the pattern, the image shown was averaged for 10 min to enhance the contrast. (d) Fluorescent control image of an ultramicrotome sectioned HeLa cell $(150 \mathrm{~nm})$, where the plasma membrane was labelled with biotin-poly-L-lysine-Gd ${ }^{3+}$-DTPA-Alexa532 (Alexa532 fluorescence spectrally filtered from $550-575 \mathrm{~nm}$ ). (e) Magnetic imaging via a single- $\tau$ measurement $\left(t_{\mathrm{m}}=15 \mathrm{~min}, \tau=440 \mu \mathrm{s}, B_{0} \sim 50 \mu \mathrm{T}\right)$ evidencing the presence of magnetic $\mathrm{Gd}^{3+}$ at the cell membrane (dark structures). (f) Line scan through plasma membrane shown in (e) demonstrating spatial resolution of $472 \mathrm{~nm}$. Errors bars, $1 \sigma$ s.e. of six independent line scans. Scale bars, $5 \mu \mathrm{m}$.

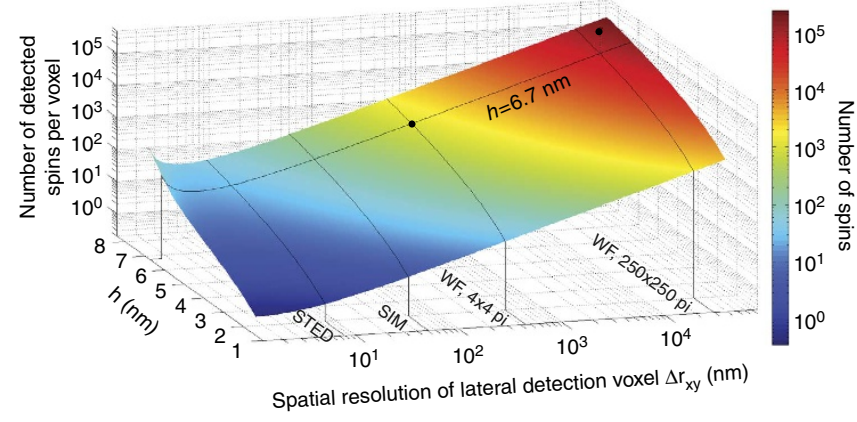

Figure 4 | Sensitivities of optimized single- $\tau$ detection. The minimum number of detectable spins $n_{\mathrm{Gd}}$ was simulated for $t_{\mathrm{m}}=20 \mathrm{~s}$ as a function of sample-sensor distance $h$ and spatial resolution $\Delta r_{x y}$. Experimental results with $h=6.7 \mathrm{~nm}$ and two distinct pixel binnings (black dots, WF: widefield detection) demonstrate good agreement. Fewer spins per voxel can be detected by decreasing $h$ and/or the detection voxel equivalent to an increase in spatial resolution. Note that $T_{1, \text { int }}$ remains to be experimentally determined for shallow NV depths with $h<5 \mathrm{~nm}$ and is assumed to be constant for the simulation.

optimal NV readout with subsequent re-polarization into the $|0\rangle$ ground state enabling CCD integration over multiple repetitions $\left(N \sim 10^{4}\right.$ per image). The fluorescent NV response was spectrally filtered (LP650, Omega) and projected onto a $512 \times 512$ cooled EM-CCD (CascadeII, Roper Scientific) yielding an effective pixel size of $115 \mathrm{~nm}$. Homogeneous and resonant microwave manipulation of the NV sensor with an optical field of view of $\sim 60 \times 60 \mu \mathrm{m}^{2}$ was achieved using a lithographically grown broadband $\Omega$-shaped structure. The flow rate in the microfluidic channel made of transparent polydimethylsiloxan (PDMS Sylgard) was adjusted to $1 \mu \mathrm{s}^{-1}$. All experiments were conducted with commercially available $\mathrm{Gd}^{3+}$ solution (Gadovist, Bayer Schering Pharma), except for the spin contrast imaging of cellular structures (see cell preparation and synthesis of magnetic/fluorescent spin label in detailed method section). Alexa532 was imaged using a separate fluorescence filter (Semrock, HC 565/24).

Cell culture, labelling and preparation. In all, $2.5 \times 10^{6} \mathrm{HeLa}$ ATCC cells were harvested and suspended in PBS buffer at a concentration of $25 \times 10^{6}$ cells per ml Ten microlitre containing $20 \mathrm{mM}$ solution of EZ-Link NHS Biotin (Pierce, 20217) in dimethylsulphoxide were added for $20 \mathrm{~min}$ to the cells for biotinylation. The cells were then fixed with $4 \%$ paraformaldehyde, extensively washed and resuspended in PBS buffer. After 5 min incubation with $100 \mu \mathrm{g}$ streptavidin, cells were washed in PBS buffer, resuspended and incubated for $5 \mathrm{~min}$ with $\sim 1 \mathrm{nmol}$ of biotin-poly-L-lysine Gd-DTPA (see synthesis below). After extensive washing with PBS Puffer, cells were resuspended in $1 \mathrm{ml}$ of $1.25 \mathrm{M}$ sucrose solution and harvested by centrifugation. The cell pellets were mounted on an aluminium stub and vitrified by submersion in liquid nitrogen. Then, thin cell sections with a thickness of $\sim 150 \mathrm{~nm}$ were produced with an Ultramicrotome (Leica UCT) equipped with a LKB Cryokit and freshly prepared glass knives (Leica KMR2). Frozen cell cuts were transferred onto the diamond under ambient conditions and embedded in PDMS to minimize changes in the refractive index.

Synthesis of Biotin-poly-L-lysine-Gd ${ }^{3+}$-DTPA-Alexa532. Twenty-five microgram poly-L-lysine (Sigma-Aldrich, P2636) was dissolved in $0.1 \mathrm{M}$ sodium hydrogencarbonate buffer (pH8.3). Then, $600 \mu$ mol diethylenetriaminepentaaceticdianhydride (Sigma-Aldrich, 284025) was added slowly while stirring and regulating to $\mathrm{pH} 8$. The solution was stirred for $2 \mathrm{~h}$ at $4{ }^{\circ} \mathrm{C}$ and was extensively dialyzed against $0.1 \mathrm{M}$ sodium hydrogencarbonate using a ZelluTrans dialysis membrane 
(Carl Roth, E671.1). The obtained poly-L-lysine-DTPA was biotinylated by addition of $3 \mu \mathrm{mol} \mathrm{EZ-Link} \mathrm{NHS-Biotin.} \mathrm{After} 2 \mathrm{~h}$ of stirring at $4{ }^{\circ} \mathrm{C}$ the solution was dialyzed against $1 \mathrm{M}$ citrate buffer (pH6.5). Six hundred micromol gadolinium chloride hexahydrate (Sigma-Aldrich, G7532) was dissolved in $0.1 \mathrm{M}$ sodium acetate (pH6) and slowly added to the biotin-poly-L-lysine-DTPA solution. After $20 \mathrm{~h}$ of stirring at $4^{\circ} \mathrm{C}$, the solution was first dialyzed against $1 \mathrm{M}$ citrate buffer (pH6.5) to remove free $\mathrm{Gd}^{3+}$ ions within the solution followed by a dialysis against $0.1 \mathrm{M}$ sodium hydrogencarbonat buffer. Then, $1 \mathrm{mg}$ Alexa532 carboxylicacid-succinimidylester (Invitrogen, A-2010) was dissolved in $200 \mu \mathrm{l}$ of dimethylsulphoxide and slowly added to the biotin-poly-L-lysine-Gd ${ }^{3}{ }^{+}$-DTPA solution. After stirring at $4{ }^{\circ} \mathrm{C}$ for $30 \mathrm{~min}$, the free dye was removed while simultaneously concentrating the labelled probe using a Centriprep Ultracel (Millipore, YM.10).

$\boldsymbol{T}_{1}$-decay fitting. Recorded $T_{1}$ curves were fit with a bi-exponential function $I(\tau)=I_{0}+A_{\text {short }} \cdot \exp \left(\tau / T_{1, \text { short }}\right)+A_{\text {long }} \cdot \exp \left(\tau / T_{1, \text { long }}\right)$, as the decay exhibited two components with $T_{1 \text {,short }}<<T_{1, \text { long and }} A_{\text {long }}>A_{\text {short }}$. We attribute the shorter component $\left(T_{1, \text { short }}<15 \mu \mathrm{s}\right)$ to a few clustered and thus very proximal NV pairs subjected to cross-relaxation. The relaxation rates $\Gamma_{1}$ and the corresponding relaxation times $T_{1}$ given in the text are determined by the main decay of the second and significantly longer $T_{1}$ component.

\section{References}

1. Bloch, F. Nuclear induction. Phys. Rev 70, 460-474 (1946).

2. Clarke, J. \& Braginski, A. I. The SQUID Handbook: Applications of SQUIDs and SQUID systems (Wiley-VCH, 2006).

3. Rugar, D., Budakian, R., Mamin, H. J. \& Chui, B. W. Single spin detection by magnetic resonance force microscopy. Nature 430, 329-332 (2004).

4. Budker, D. \& Romalis, M. Optical magnetometry. Nat. Phys. 3, 227-234 (2007).

5. Bajaj, V. S., Paulsen, J., Harel, E. \& Pines, A. Zooming in on microscopic flow by remotely detected MRI. Science 330, 1078-1081 (2010).

6. Gruber, A. et al. Scanning confocal optical microscopy and magnetic resonance on single defect centers. Science 276, 2012-2014 (1997).

7. Chernobrod, B. M. \& Berman, G. P. Spin microscope based on optically detected magnetic resonance. J. Appl. Phys. 97, 014903 (2005).

8. Degen, C. Nanoscale magnetometry: microscopy with single spins. Nat. Nano 3, 643-644 (2008)

9. Taylor, J. M. et al. High-sensitivity diamond magnetometer with nanoscale resolution. Nat. Phys. 4, 810-816 (2008).

10. Maze, J. R. et al. Nanoscale magnetic sensing with an individual electronic spin in diamond. Nature 455, 644-647 (2008).

11. Balasubramanian, G. et al. Ultralong spin coherence time in isotopically engineered diamond. Nat. Mater. 8, 383-387 (2009).

12. Steinert, S. et al. High sensitivity magnetic imaging using an array of spins in diamond. Rev. Sci. Instr. 81, 043705 (2010).

13. Pham, L. M. et al. Magnetic field imaging with nitrogen-vacancy ensembles. N. J. Phys. 13, 045021 (2011).

14. Maletinsky, P. et al. A robust scanning diamond sensor for nanoscale imaging with single nitrogen-vacancy centres. Nat. Nanotechnol. 7, 320-324 (2012).

15. Neumann, P. et al. Quantum register based on coupled electron spins in a room-temperature solid. Nat. Phys. 6, 249-253 (2010).

16. Zhao, N. et al. Sensing single remote nuclear spins. Nat. Nanotechnol. 7, 657-662 (2012).

17. Kolkowitz, S., Unterreithmeier, Q. P., Bennett, S. D. \& Lukin, M. D. Sensing distant nuclear spins with a single electron spin. Phys. Rev. Lett. 109, 137601 (2012).

18. Taminiau, T. H. et al. Detection and control of individual nuclear spins using a weakly coupled electron spin. Phys. Rev. Lett. 109, 137602 (2012).

19. Grotz, B. et al. Sensing external spins with nitrogen-vacancy diamond. N. J. Phys. 13, 055004 (2011).

20. Cole, J. H. \& Hollenberg, L. C. L. Scanning quantum decoherence microscopy. Nanotechnology 20, 495401 (2009).

21. Hall, L. T., Cole, J. H., Hill, C. D. \& Hollenberg, L. C. L. Sensing of fluctuating nanoscale magnetic fields using nitrogen-vacancy centers in diamond. Phys. Rev. Lett. 103, 220802 (2009).

22. Taylor, J. M. et al. High-sensitivity diamond magnetometer with nanoscale resolution. Nat. Phy. 4, 810 (2008).

23. Acosta, V. M. et al. Diamonds with a high density of nitrogen-vacancy centers for magnetometry applications. Phys. Rev. B 80, 115202 (2009).

24. Ziegler, J. F. The Stopping and Range of Ions in Solids (Pergamon. P.: 1985).

25 . Du, J. et al. Preserving electron spin coherence in solids by optimal dynamical decoupling. Nature 461, 1265-1268 (2009).
26. de Lange, G., Wang, Z. H., Ristè, D., Dobrovitski, V. V. \& Hanson, R. Universal dynamical decoupling of a single solid-state spin from a spin bath. Science 330, 60-63 (2010).

27. Hall, L. T., Hill, C. D., Cole, J. H. \& Hollenberg, L. C. L. Ultrasensitive diamond magnetometry using optimal dynamic decoupling. Phys. Rev. B 82, 045208 (2010).

28. Chan, K. \& Wong, W. Small molecular gadolinium(III) complexes as MRI contrast agents for diagnostic imaging. Coord. Chem. Rev. 251, 2428-2451 (2007).

29. Kruk, D., Kowalewski, J. \& Westlund, P.-O. Nuclear and electron spin relaxation in paramagnetic complexes in solution: effects of the quantum nature of molecular vibrations. J. Chem. Phys. 121, 2215 (2004).

30. Jarmola, A., Acosta, V. M., Jensen, K., Chemerisov, S. \& Budker, D. Temperature- and magnetic-field-dependent longitudinal spin relaxation in nitrogen-vacancy ensembles in diamond. Phys. Rev. Lett. 108, 197601 (2012).

31. Dröge, W. Free radicals in the physiological control of cell function. Physiol. Rev. 82, 47-95 (2002).

32. Lovell, M., Robertson, J., Teesdale, W., Campbell, J. \& Markesbery, W. Copper, iron and zinc in Alzheimer's disease senile plaques. J. Neurol. Sci. 158, 47-52 (1998).

33. Müller, N. \& Jerschow, A. Nuclear spin noise imaging. PNAS 103, 6790-6792 (2006).

34. Blank, A., Dunnam, C. R., Borbat, P. P. \& Freed, J. H. Pulsed three-dimensional electron spin resonance microscopy. Appl. Phys. Lett. 85, 5430-5432 (2004).

35. Crooker, S. A., Rickel, D. G., Balatsky, A. V. \& Smith, D. L. Spectroscopy of spontaneous spin noise as a probe of spin dynamics and magnetic resonance. Nature 431, 49-52 (2004).

36. Degen, C. L., Poggio, M., Mamin, H. J., Rettner, C. T. \& Rugar, D. Nanoscale magnetic resonance imaging. Proc. Natl Acad. Sci. 106, 1313-1317 (2009).

37. Gustafsson, M. G. L. Nonlinear structured-illumination microscopy: Wide-field fluorescence imaging with theoretically unlimited resolution. Proc. Natl Acad. Sci. USA 102, 13081-13086 (2005).

38. Rittweger, E., Han, K. Y., Irvine, S. E., Eggeling, C. \& Hell, S. W. STED microscopy reveals crystal colour centres with nanometric resolution. Nat. Photon 3, 144-147 (2009).

39. McGuinness, L. P. et al. Quantum measurement and orientation tracking of fluorescent nanodiamonds inside living cells. Nat. Nanotechnol. 6, 358-363 (2011).

40. McGuinness, L. P. et al. Ambient Nanoscale Sensing with Single Spins Using Quantum Decoherence. arXiv:1211.5749 (2012); at http://arxiv.org/abs/ 1211.5749.

41. Hall, L. T. et al. Monitoring ion-channel function in real time through quantum decoherence. Proc. Natl. Acad. Sci. 107, 18777-18782 (2010).

\section{Acknowledgements}

This work was supported by Volkswagen Stiftung, Baden Württemberg Stiftung, DARPA via project QUASAR, the EU Projects Dinamo and SQUTEC, the German science foundation via the research group 1493 and the Australian Research Council Centre of Excellence for Quantum Computation and Communication Technology (CE1 10001027) We thank Chang Shin and Rainer Stöhr for technical assistance with the resonator and the optical photolithography, respectively, and Friedemann Reinhard, Fedor Jelezko, Liam McGuiness and Phil Hemmer for helpful discussions.

\section{Author contributions}

S.S., G.B. and J.W. conceived the study; S.S. and F.Z. performed experiments and carried out analysis of the data. A.Z. and M.S. prepared the labelled cell sections. L.T.H., S.S. and L.H. developed the theory. All authors discussed the results and participated in writing the manuscript.

\section{Additional information}

Supplementary Information accompanies this paper at http://www.nature.com/ naturecommunications

Competing financial interests: The authors declare no competing financial interests.

Reprints and permission information is available online at http://npg.nature.com/ reprintsandpermissions/

How to cite this article: Steinert, S. et al. Magnetic spin imaging under ambient conditions with sub-cellular resolution. Nat. Commun. 4:1607 doi: 10.1038/ncomms2588 (2013). 